\title{
Effects of Linear Central Potential Induced by Lorentz Symmetry Violation framework and Cornell-type Non-electromagnetic Potential on Spin-0 Scalar Particles
}

\author{
Faizuddin Ahmed ${ }^{1}$ \\ National Academy Gauripur, Assam, 783331, India
}

\begin{abstract}
The relativistic quantum dynamics of a spin-0 scalar particle under the effects of the violation of Lorentz symmetry in the presence of a non-electromagnetic potential is analyzed. The central potential induced by the Lorentz symmetry violation is a linear electric and constant magnetic field and, analyze the effects on the eigenvalues and the wave function. We see there is a dependence of the linear charge density on the quantum numbers of the system.
\end{abstract}

Keywords: Lorentz symmetry violation, Relativistic wave equations, non-electromagnetic potential, electric \& magnetic field, biconfluent Heun's function .

PACS Number(s): 11.30.Qc, 03.65.Pm, 11.30.Cp

\section{Introduction}

The Standard Model extension (SME) $[1,2,3,4]$ is the framework for investigating signals of Lorentz violation and studying properties of physical systems as it includes Lorentz violating terms in all sectors of the minimal Standard Model. The Lorentz-violating terms are generated as vacuum expectation values of tensors quantities, keeping the coordinate invariance of

\footnotetext{
${ }^{1}$ faizuddinahmed15@gmail.com ; faiz4U.enter@rediffmail.com
} 
the extended theory [5]. This model has been scrutinized in many respects with the studies of fermion and gauge sectors, and gravitation extension $[6,7,8,9,10,11,12,13,14,15]$, fermion systems and radiative corrections $[16,17,18,19,20,21,22,23,24,25,26]$, CPT-probing experiments $[27,28,29,30,31,32]$, the electromagnetic CPT- and Lorentz-odd term $[33,34,35,36,37,38,39,40,41,42,43,44,45,46]$, and electromagnetic CPT-even and Lorentz-odd coefficients [47, 48, 49, 50, 51, 52, 53].

In this paper, we study motion of a relativistic scalar particle under Lorentz symmetry breaking defined by a tensor $\left(K_{F}\right)_{\mu \nu \alpha \beta}$ out of the Standard Model Extension (SME) in the presence of a non-electromagnetic potential. We investigate the effects of a linear central potential induced by Lorentz symmetry violation and the solution of the bound state to the Klein-Gordon equation can be achieved. The study of a relativistic scalar particle under the influence of a non-electromagnetic potential (here we choose a Cornell-type potential) has been of growing interest in different branches of physics. Several authors have been studied the relativistic quantum systems in the presence of various kind of electromagnetic and non-electromagnetic potentials under the effects of Lorentz symmetry violation $[1,54,55,56,57,58,59]$. Noted that the gauge sector of Standard Model Extension consists of two classes that modifies the transport properties of space-time. These two classes are called the CPT-odd sector [1] and the CPT-even sector [43, 60].

In the proposed work, we have chosen the Cornell-type potential which consist of two concepts-confinement and asymptotic freedom. The Coulomblike part is responsible for the interactions at short ranges whereas, and linear potential term lead to the confinement quarks phenomena. This type of potential has been used to study the relativistic quantum system [61, 62, $63,64,65,66,67,68,69,70,71]$, bound states of hadrons [72, 73], and the ground state of three quarks [74] in particle physics. The Cornell-type potential in cylindrical system is given by

$$
S(r)=\eta_{L} r+\frac{\eta_{c}}{r}
$$


where $\eta_{L}>0, \eta_{c}>0$ are arbitrary constants.

The quantum dynamics of spin-0 scalar particle under the effects of Lorentz symmetry violation is $[1,54,55,56,57,58,59,63,66,71,75,76,77]$

$$
p^{\mu} p_{\mu} \Psi+\frac{\alpha}{4}\left(K_{F}\right)_{\mu \nu \alpha \beta} F^{\mu \nu}(x) F^{\alpha \beta}(x) \Psi=(M+S)^{2} \Psi
$$

where $\alpha$ is a constant, $F_{\mu \nu}(x)$ is the electromagnetic field tensor, $\left(K_{F}\right)_{\mu \nu \alpha \beta}$ is a dimensionless tensor that governs the Lorentz symmetry violation out of the Standard Model Extension, and $S(r)$ is the non-electromagnetic potential.

\section{Relativistic Scalar particle under the ef- fects of LSV with a non-electromagnetic Potential}

The Klein-Gordon equation under the effects of Lorentz symmetry violation defined by the Eq. (2) in the background of the Minkowski space-time is given by

$$
\begin{aligned}
& {\left[-\frac{\partial^{2}}{\partial t^{2}}+\frac{\partial^{2}}{\partial r^{2}}+\frac{1}{r} \frac{\partial}{\partial r}+\frac{\partial^{2}}{\partial z^{2}}+\frac{1}{r^{2}} \frac{\partial^{2}}{\partial \phi^{2}}\right] \Psi+\frac{\alpha}{4}\left(K_{F}\right)_{\mu \nu \alpha \beta} F^{\mu \nu}(x) F^{\alpha \beta}(x) \Psi} \\
& =(M+S)^{2} \Psi .
\end{aligned}
$$

Using the properties of the tensor $\left(K_{F}\right)_{\mu \nu \alpha \beta}[1,2,3,4,54,55,56,57,58$, $59,75,76]$, we can rewrite (3) in the following form :

$$
\begin{aligned}
& {\left[-\frac{\partial^{2}}{\partial t^{2}}+\frac{\partial^{2}}{\partial r^{2}}+\frac{1}{r} \frac{\partial}{\partial r}+\frac{1}{r^{2}} \frac{\partial^{2}}{\partial \phi^{2}}+\frac{\partial^{2}}{\partial z^{2}}\right] \Psi} \\
& +\left[-\frac{\alpha}{2}\left(\kappa_{D E}\right)_{i j} E^{i} E^{j}+\frac{\alpha}{2}\left(\kappa_{H B}\right)_{j k} B^{i} B^{j}-\alpha\left(\kappa_{D B}\right)_{j k} E^{i} B^{j}\right] \Psi \\
& =(M+S)^{2} \Psi .
\end{aligned}
$$

We choose the scenario of the Lorentz symmetry violation determined by the non-null components $\left(\kappa_{D E}\right)_{11}=$ const $=\kappa_{1},\left(\kappa_{H B}\right)_{33}=$ const $=\kappa_{2}$ and 
$\left(\kappa_{D B}\right)_{13}=$ const $=\kappa_{3}$ with the following field configuration $[57,58,59,63$, $75,76,77,78,79]$ :

$$
\vec{B}=B_{0} \hat{z} \quad, \quad \vec{E}=\frac{\lambda}{2} r \hat{r}
$$

where $B_{0}>0, \hat{z}$ is a unit vector in the $z$-direction, $\lambda$ is a constant associated with a linear charge density of electric charges along the axial direction, and $\hat{r}$ is the unit vector in the radial direction.

Hence, equation (4) using the configuration (5) becomes

$$
\begin{aligned}
& {\left[-\frac{\partial^{2}}{\partial t^{2}}+\frac{\partial^{2}}{\partial r^{2}}+\frac{1}{r} \frac{\partial}{\partial r}+\frac{1}{r^{2}} \frac{\partial^{2}}{\partial \phi^{2}}+\frac{\partial^{2}}{\partial z^{2}}\right] \Psi} \\
& +\left[-\frac{\alpha \kappa_{1} \lambda^{2}}{8} r^{2}+\frac{\alpha \kappa_{2} B_{0}^{2}}{2}-\frac{\alpha \lambda B_{0} \kappa_{3}}{2} r\right] \Psi=(M+S)^{2} \Psi .
\end{aligned}
$$

Since the metric is independent of $(t, \phi, z)$, let the solution to the Eq. (7) is

$$
\Psi(t, r, \phi, z)=e^{i(-E t+l \phi+k z)} \psi(r),
$$

where $E, l$ have their usual meaning, and $k$ is a constant.

Therefore substituting the potential (1) into the Eq. (6) and using (7), we obtain the following radial wave equation for $\psi(r)$ :

$$
\psi^{\prime \prime}(r)+\frac{1}{r} \psi^{\prime}(r)+\left[\Sigma-\frac{j^{2}}{r^{2}}-\omega^{2} r^{2}-\frac{\beta_{1}}{r}-\beta_{2} r\right] \psi(r)=0,
$$

where

$$
\begin{aligned}
\Sigma & =E^{2}-M^{2}-k^{2}-2 \eta_{L} \eta_{c}+\frac{1}{2} \alpha \kappa_{2} B_{0}^{2}, \\
j & =\sqrt{l^{2}+\eta_{c}^{2}}, \\
\omega & =\sqrt{\frac{1}{8} \alpha \kappa_{1} \lambda^{2}+\eta_{L}^{2}}, \\
\beta_{1} & =2 M \eta_{c}, \\
\beta_{2} & =2 M \eta_{L}+\frac{1}{2} \alpha \lambda B_{0} \kappa_{3} .
\end{aligned}
$$

Transforming $\xi=\sqrt{\omega} r$ in the above equation (8), we have

$$
\left[\frac{d^{2}}{d \xi^{2}}+\frac{1}{\xi} \frac{d}{d \xi}+\zeta-\xi^{2}-\frac{j^{2}}{\xi^{2}}-\frac{\eta}{\xi}-\theta \xi\right] \psi(\xi)=0
$$


where

$$
\zeta=\frac{\Sigma}{\omega} \quad, \quad \eta=\frac{\beta_{1}}{\sqrt{\omega}} \quad, \quad \theta=\frac{\beta_{2}}{\omega^{\frac{3}{2}}} .
$$

Now we impose the requirement that the wave-function $\psi(x)$ is finite both at the origin $x \rightarrow 0$ and at $x \rightarrow \infty$. Suppose the possible solution to the Eq. (10) is

$$
\psi(\xi)=\xi^{j} e^{-\frac{1}{2}(\xi+\theta) \xi} H(\xi) .
$$

Substituting the solution (12) into the Eq. (10), we obtain the following equation

$$
H^{\prime \prime}(\xi)+\left[\frac{1+2 j}{\xi}-2 \xi-\theta\right] H^{\prime}(\xi)+\left[-\frac{\tau}{\xi}+\Theta\right] H(\xi)=0,
$$

where

$$
\Theta=\zeta+\frac{\theta^{2}}{4}-2(1+j) \quad, \quad \tau=\eta+\frac{\theta}{2}(1+2 j) .
$$

Equation (13) is the biconfluent Heun's differential equation [61, 62, 64, 65, $67,68,69,70,80,81]$ with $H(\xi)$ is the Heun polynomials function.

The above equation (13) can be solved by the Frobenius method. Writing the solution as a power series expansion around the origin [82]:

$$
H(\xi)=\sum_{i=0}^{\infty} d_{i} \xi^{i}
$$

Substituting the power series solution into the Eq. (15), we obtain the following recurrence relation

$$
d_{n+2}=\frac{1}{(n+2)(n+2+2 j)}\left[\left\{\eta+\theta\left(n+\frac{3}{2}+j\right)\right\} d_{n+1}-(\Theta-2 n) d_{n}\right] .
$$

With the few coefficients are

$$
\begin{aligned}
& d_{1}=\left(\frac{\eta}{1+2 j}+\frac{\theta}{2}\right) d_{0} \\
& d_{2}=\frac{1}{4(1+j)}\left[\left(\eta+\theta\left(j+\frac{3}{2}\right)\right) d_{1}-\Theta d_{0}\right] .
\end{aligned}
$$


The power series expansion $H(\xi)$ becomes a polynomial of degree $n$ by imposing the following two conditions $[61,62,64,65,67,68,69,70]$

$$
\begin{aligned}
\Theta & =2 n, \quad(n=1,2, \ldots) \\
d_{n+1} & =0 .
\end{aligned}
$$

By analyzing the first condition, we obtain following equation of the energy eigenvalue $E_{n, l}$ :

$$
E_{n, l}^{2}=M^{2}+k^{2}+2 \eta_{L} \eta_{c}+2 \omega\left(n+1+\sqrt{l^{2}+\eta_{c}^{2}}\right)-\frac{1}{2} \alpha B_{0}^{2} \kappa_{2}-\frac{\beta_{2}^{2}}{4 \omega^{2}} .
$$

It is worth mentioning that we have adjusted the parameters such that $\left(M^{2}+k^{2}+2 \eta_{L} \eta_{c}+2 \omega\left(n+1+\sqrt{l^{2}+\eta_{c}^{2}}\right)\right)>\left(\frac{1}{2} \alpha B_{0}^{2} \kappa_{2}+\frac{a_{2}^{2}}{4 \omega^{2}}\right)$ and thus, the energy eigenvalue is real. Note that the Eq. (19) is not the general expression of energy eigenvalue of a relativistic scalar particle. One can obtain the individual energy level and the eigenfunction one by one by imposing the additional recurrence condition $d_{n+1}=0$ on the eigenvalue problem.

The corresponding wave-functions are given by

$$
\psi_{n, l}(\xi)=\xi^{\sqrt{l^{2}+\eta_{c}^{2}}} e^{-\frac{1}{2}\left[\xi+\frac{\beta_{2}}{\omega^{\frac{3}{2}}}\right] \xi} H(\xi) .
$$

Now, we evaluate the individual energy levels and the eigenfunctions one by one as done in [61]. For example, $n=1$, we have $\Theta=2$ and $d_{2}=0$ which implies

$$
\begin{aligned}
& \Rightarrow \frac{2}{\eta+\theta\left(\frac{3}{2}+j\right)} d_{0}=\left(\frac{\eta}{1+2 j}+\frac{\theta}{2}\right) d_{0} \\
& \Rightarrow\left(\beta_{2}^{1, l}\right)^{2}+8 \beta_{1} \beta_{2}^{1, l} \omega\left(\frac{1+j}{(1+2 j)(3+2 j)}\right)+\left[\frac{4 \beta_{1}^{2} \omega^{2}}{(1+2 j)(3+2 j)}-\frac{8 \Omega^{3}}{(3+2 j)}\right]=0 \\
& \Rightarrow \beta_{2}^{1, l}=\frac{1}{2}\left[-\frac{4 \beta_{1} \omega(1+j)}{(1+2 j)(3+2 j)}+4 \omega \sqrt{\frac{\beta_{1}^{2}}{4(1+2 j)^{2}(3+2 j)^{2}}+\frac{2}{(3+2 j)}}\right] \\
& =\frac{2 \omega}{(1+2 j)(3+2 j)}\left[-\beta_{1}(1+j)+\sqrt{\frac{\beta_{1}^{2}}{4}+2(1+2 j)^{2}(3+2 j)}\right]
\end{aligned}
$$


a constraint on the parameter $\beta_{2}$, that is, on the magnetic field $B_{0}$. We can see from Eq. (21), that the allowed values of the the magnetic field depends on the quantum numbers of the system $\{n, l\}$, the parameters associated with the background of the Lorentz Symmetry Violation, and the potential parameters.

The allowed values of the the magnetic field for the radial mode $n=1$ is

$$
B_{0}^{1, l}=\frac{\beta_{2}^{1, l}-2 M \eta_{L}}{\frac{1}{2} \alpha \lambda \kappa_{3}}
$$

Therefore, the ground state energy level for the radial mode $n=1$ is given by

$E_{1, l}= \pm \sqrt{M^{2}+k^{2}+2 \eta_{L} \eta_{c}+2 \omega\left(2+\sqrt{l^{2}+\eta_{c}^{2}}\right)-\frac{1}{2} \alpha\left(B_{0}^{1, l}\right)^{2} \kappa_{2}-\left(\frac{\beta_{2}^{1, l}}{2 \omega}\right)^{2}}$.

And the ground state eigenfunction is

$$
\psi_{1, l}(\xi)=\xi^{\sqrt{l^{2}+\eta_{c}^{2}}} e^{-\frac{1}{2}\left[\xi+\frac{\beta_{2}^{1, l}}{\Omega^{\frac{3}{2}}}\right] \xi}\left(1+d_{1} \xi\right),
$$

where we have chosen $d_{0}=1$ and

$$
d_{1}=\frac{1}{\sqrt{\omega}}\left[\frac{2 M \eta_{c}}{\left(1+2 \sqrt{l^{2}+\eta_{c}^{2}}\right)}+\frac{\beta_{2}^{1, l}}{2 \omega}\right]
$$

We can see that the lowest energy state (23) plus the ground state wave function (24)-(25) is defined for the radial mode $n=1$, instead of $n=0$. This effect arises due to the presence of Cornell-type potential and the Lorentz symmetry violation in the relativistic quantum system.

\section{Conclusions}

We have investigated the effects of a linear central potential induced by the violation of Lorentz symmetry breaking background on a relativistic scalar 
particle. Despite being inspired by the CPT-even sector of the SME, our proposal is not inserted in the scenario of Standard Model Extension (SME), which can be promising because it can open new discussions about new theories that covers energy scales beyond the SME. Since terms that violate the Lorentz symmetry start with tiny values and go for energy scales beyond the Standard Model Extension, we have relaxed the renormalization property of our model. In this work, we have shown that the bound states solution to the Klein-Gordon equation can be obtained in the scenario of Lorentz symmetry breaking effects defined by a radial linear electric field produced by a linear distribution of electric charges, a uniform magnetic along the $z$-direction, a tensor background that governs the Lorentz symmetry violation possessing non-null components $\left(\kappa_{D B}\right)_{13}=$ const, $\left(\kappa_{D E}\right)_{11}=$ const and $\left(\kappa_{H B}\right)_{33}=$ const subject to a Cornell-type scalar potential. We have obtained the non-compact expression of the energy eigenvalues (19) and the wave-function (20). By imposing the additional recurrence condition $d_{n+1}=0$ on the eigenvalue problem, one can obtain the individual energy levels and wave-function, for example, we have obtained the lowest state energy level (22) and the corresponding wave-function (23)-(24) for the radial mode $n=1$ instead of $n=0$. This effect arises due to the presence of the Cornell-type scalar potential and Lorentz symmetry violation background. We have seen a quantum effect due to the dependence of the magnetic field $B_{0}$ on the quantum numbers $\{n, l\}$ of the relativistic quantum system. We can see in both case that the energy level and the wave-function get modified due to the presence of the tensor $\left(K_{F}\right)_{\mu \nu \alpha \beta}$ that governs the Lorentz symmetry violation and the potential parameters.

\section{Conflict of Interest}

There is no conflict of interest regarding publication this paper. 


\section{Data Availability}

No data has been used to prepare this paper.

\section{References}

[1] D. Colladay and V. A. Kostelecky, Phys. Rev. D 55, 6760 (1997); ibid 58, 116002 (1998).

[2] S. R. Coleman and S. L. Glashow, Phys. Rev. D 59, 116008 (1999).

[3] V. A. Kostelecky and S. Samuel, Phys. Rev. Lett. 63, 224 (1989); ibid 66, 1811 (1991); Phys. Rev. D 39, 683 (1989); ibid 40, 1886 (1989).

[4] V. A. Kostelecky and R. Potting, Nucl. Phys. B 359, 545 (1991); Phys. Lett. B 381, 89 (1996); Phys. Rev. D 51, 3923 (1995).

[5] V. A. Kostelecky and R. Lehnert, Phys. Rev. D 63, 065008 (2001).

[6] V. A. Kostelecky, Phys. Rev. D 69, 105009 (2004);

[7] V. A. Kostelecky, Neil Russell, and J. D. Tasson, Phys. Rev. Lett. 100, 111102 (2008);

[8] V. A. Kostelecky and J. D. Tasson, Phys. Rev. Lett. 102, 010402 (2009).

[9] Q. G. Bailey and V. A. Kostelecky, Phys. Rev. D 74, 045001 (2006).

[10] Q. G. Bailey, Phys. Rev. D 80, 044004 (2009).

[11] V. A. Kostelecky and R. Potting, Phys. Rev. D 79, 065018 (2009).

[12] Q. G. Bailey, Phys. Rev. D 82, 065012 (2010).

[13] V. B. Bezerra, C. N. Ferreira, and J. A. Helayel-Neto, Phys. Rev. D 71, 044018 (2005). 
[14] J. L. Boldo, J. A. Helayel-Neto, L. M. de Moraes, C. A. G. Sasaki, and V. J. V. Otoya, Phys. Lett. B 689, 112 (2010).

[15] V. A. Kostelecky and J. D. Tasson, Phys. Rev. D 83, 016013 (2011).

[16] B. Altschul, Phys. Rev. D 70, 056005 (2004).

[17] G. M. Shore, Nucl. Phys. B 717, 86 (2005).

[18] D. Colladay and V. A. Kostelecky, Phys. Lett. B 511, 209 (2001).

[19] O. G. Kharlanov and V. Ch. Zhukovsky, J. Math. Phys. (N. Y.) 48, 092302 (2007).

[20] R. Lehnert, Phys. Rev. D 68, 085003 (2003).

[21] V. A. Kostelecky and C. D. Lane, J. Math. Phys. (N. Y.) 40, 6245 (1999).

[22] R. Lehnert, J. Math. Phys. (N. Y.) 45, 3399 (2004).

[23] W. F. Chen and G. Kunstatter, Phys. Rev. D 62, 105029 (2000).

[24] B. Goncalves, Y. N. Obukhov, and I. L. Shapiro, Phys. Rev. D 80, 125034 (2009).

[25] V. A. Kostelecky and R. Lehnert, Phys. Rev. D 63, 065008 (2001).

[26] S. Chen, B. Wang, and R. Su, Classical Quantum Gravity 23, 7581 (2006).

[27] R. Bluhm, V. A. Kostelecky, and N. Russell, Phys. Rev. Lett. 79, 1432 (1997); Phys. Rev. D 57, 3932 (1998).

[28] R. Bluhm, V. A. Kostelecky, C. D. Lane, and N. Russell, Phys. Rev. Lett. 88, 090801 (2002). 
[29] R. Bluhm and V. A. Kostelecky, Phys. Rev. Lett. 84, 1381 (2000).

[30] R. Bluhm, V.A. Kostelecky, and C.D. Lane, Phys. Rev. Lett. 84, 1098 (2000).

[31] R. Bluhm, V. A. Kostelecky, and N. Russell, Phys. Rev. Lett. 82, 2254 (1999).

[32] V. A. Kostelecky and C. D. Lane, Phys. Rev. D 60, 116010 (1999).

[33] C. Adam and F. R. Klinkhamer, Nucl. Phys. B 607, 247 (2001); ibid B 657, 214 (2003).

[34] A.A. Andrianov and R. Soldati, Phys. Rev. D 51, 5961 (1995); Phys. Lett. B 435, 449 (1998).

[35] A. A. Andrianov, R. Soldati, and L. Sorbo, Phys. Rev. D 59, 025002 (1998);

[36] A. A. Andrianov, D. Espriu, P. Giacconi, and R. Soldati, J. High Energy Phys. 09 (2009) 057.

[37] J. Alfaro, A. A. Andrianov, M. Cambiaso, P. Giacconi, and R. Soldati, Int. J. Mod. Phys. A 25, 3271 (2010);

[38] V. Ch. Zhukovsky, A. E. Lobanov, and E. M. Murchikova, Phys. Rev. D 73, 065016 (2006).

[39] R. Lehnert and R. Potting, Phys. Rev. Lett. 93, 110402 (2004); Phys. Rev. D 70, 125010 (2004);

[40] B. Altschul, Phys. Rev. D 75, 105003 (2007);

[41] C. Kaufhold and F.R. Klinkhamer, Nucl. Phys. B 734, 1 (2006).

[42] A. P. Baeta Scarpelli, H. Belich, J. L. Boldo, and J. A. Helayel-Neto, Phys. Rev. D 67, 085021 (2003); 
[43] A.P. Baeta Scarpelli, H. Belich, J. L. Boldo, L. P. Colatto, J. A. HelayelNeto, and A. L. M. A. Nogueira, Nucl. Phys. B, Proc. Suppl. 127, 105 (2004).

[44] M. B. Cantcheff, Eur. Phys. J. C 46, 247 (2006);

[45] J. A. de Sales, T. CostaSoares, and V. J. Vasquez Otoya, Physica (Amsterdam) 391A, 5422 (2012);

[46] O. M. D Cima, D. H. T. Franco, A. H. Gomes, J. M. Fonseca, and O. Piguet, Phys. Rev. D 85, 065023 (2012).

[47] V. A. Kostelecky and M. Mewes, Phys. Rev. Lett. 87, 251304 (2001); Phys. Rev. D 66, 056005 (2002); Phys. Rev. Lett. 97, 140401 (2006).

[48] F. R. Klinkhamer and M. Risse, Phys. Rev. D 77, 016002 (2008); ibid 77, 117901 (2008).

[49] F. R. Klinkhamer and M. Schreck, Phys. Rev. D 78, 085026 (2008).

[50] B. Altschul, Phys. Rev. Lett. 98, 041603 (2007).

[51] M. Schreck, Phys. Rev. D 86, 065038 (2012).

[52] B. Altschul, Nucl. Phys. B 796, 262 (2008); Phys. Rev. Lett. 98, 041603 (2007).

[53] C. Kaufhold and F.R. Klinkhamer, Phys. Rev. D 76, 025024 (2007).

[54] V. A. Kostelecky and M. Mewes, Phys. Rev. Lett. 87, 251304 (2001).

[55] V. A. Kostelecky and M. Mewes, Phys. Rev. D 66, 056005 (2002).

[56] H. Belich, F. J. L. Leal, H. L. C. Louzada and M. T. D. Orlando, Phys. Rev. D 86, 125037 (2012).

[57] K. Bakke and H. Belich, Ann. Phys. (N. Y.) 354, 1 (2015). 
[58] K. Bakke and H. Belich, Ann. Phys. (N. Y.) 373, 115 (2016).

[59] R. L. L. Vitoria and H. Belich, Eur. Phys. J. C 78, 999 (2018).

[60] S. Carroll, G. Field and R. Jackiw, Phys. Rev. D 41, 1231 (1990).

[61] E. R. F. Medeiros and E. R. Bezerra de Mello, Eur. Phys. J. C 72, 2051 (2012).

[62] Z. Wang, Z. Long, C. Long and M. Wu, Euro. Phys. J. Plus 130, 36 (2015).

[63] F. Ahmed, DOI : 10.20944/preprints202106.0006.v1.

[64] M. Hosseini, H. Hassanabadi, S. Hassanabadi and P. Sedaghatnia, Int. J. Geom. Meths. Mod. Phys. 16, 1950054 (2019).

[65] M. de Montigny, M. Hosseinpour and H. Hassanabadi, Int. J. Mod. Phys. A 31, 1650191 (2016).

[66] F. Ahmed, DOi : 10.20944/preprints202105.0748.v1.

[67] M. S. Cunha, C. R. Muniz, H. R. Christiansen and V. B. Bezerra, Eur. Phys. J. C 76, 512 (2016).

[68] F. Ahmed, Adv. High Energy Phys. 2020, 4832010 (2020).

[69] H. Hassanabadi, E. Maghsoodi and S. Zarrinkamar, Ann. Phys. (Berlin) 525, 944 (2013).

[70] M. Hosseinpour, H. Hassanabadi and Marc de Montigny, Int. J. Geom. Meths Mod. Phys. 15, 1850165 (2018).

[71] F. Ahmed, DOi : 10.20944/preprints202105.0755.v1

[72] H. Hassanabadi and S. Rahmani, Few-Body Syst. 56, 691 (2015). 
[73] H. Hassanabadi, S. Rahmani and S. Zarrinkamar, Phys. Rev. D 90, 074024 (2014).

[74] C. Alexandrou, P. de Forcrand and O. Jahn, Nuclear Phys. B (Proc. Supp.) 119, 667 (2003).

[75] M. Ericsson and E. Sjoqvist, Phys. Rev. A 65, 013607 (2001).

[76] I. C. Fonseca and K. Bakke, J. Math. Phys. 56, 062107 (2015).

[77] F. Ahmed, IJMPA (2021), DOI:10.1142/S0217751X21501281.

[78] F. Ahmed, DOI : 10.20944/preprints202106.0003.v1.

[79] F. Ahmed, DOI : 10.20944/preprints202105.0782.v1.

[80] A. Ronveaux, Heun's Differential Equations, Oxford University Press, Oxford ( 1995).

[81] S. Y. Slavyanov and W. Lay, Special Functions: A Unified Theory Based in Singularities, Oxford University Press, New York (2000).

[82] G. B. Arfken and H. J. Weber, Mathematical Methods For Physicists, Elsevier Academic Press, London (2005). 